\title{
A mapping theorem for topological complexity
}

\author{
MARK GRANT \\ GREGORY LUPTON \\ JOHN OPREA
}

\begin{abstract}
We give new lower bounds for the (higher) topological complexity of a space in terms of the Lusternik-Schnirelmann category of a certain auxiliary space. We also give new lower bounds for the rational topological complexity of a space, and more generally for the rational sectional category of a map, in terms of the rational category of a certain auxiliary space. We use our results to deduce consequences for the global (rational) homotopy structure of simply connected hyperbolic finite complexes.
\end{abstract}

55M30, 55P62; 55S40, 55Q15

\section{Introduction}

The topological complexity of a space is a numerical homotopy invariant of LusternikSchnirelmann type introduced by Farber [7] and motivated by the motion planning problem from the field of topological robotics. In this paper, we prove several results that establish new lower bounds for (higher) topological complexity in both the rational and the integral (ordinary) settings. We begin with an outline of our main results.

By cat $(X), \operatorname{secat}(p), \mathrm{TC}(X)$ and - for $n \geq 3-\mathrm{TC}_{n}(X)$, respectively, we denote the Lusternik-Schnirelmann category of a space $X$, the sectional category of a fibration $p: E \rightarrow B$, the topological complexity of $X$, and the higher topological complexity of $X$, respectively. We adopt the notational convention that $\mathrm{TC}(X)=\mathrm{TC}_{2}(X)$. We refer to Cornea et al. [4] for a general introduction to LS category and related topics, including sectional category; basic definitions are reviewed towards the end of this introduction. The original articles that introduce (higher) topological complexity, Farber [7] and Rudyak [22], discuss the connection to the motion planning problem.

Our first main result is as follows.

Theorem 1 (See Theorem 2.2) Consider two maps $f_{j}: Y_{j} \rightarrow X$ of connected spaces, $j=1,2$, with each $\left(f_{j}\right)_{\#}: \pi_{i}\left(Y_{j}\right) \rightarrow \pi_{i}(X)$ an inclusion and such that the image subgroups $\left(f_{j}\right)_{\#}\left(\pi_{i}\left(Y_{j}\right)\right)$ are complementary subgroups of $\pi_{i}(X)$ for all $i \geq 1$; see Section 2 for details. Then for $n \geq 2$ we have

$$
\operatorname{cat}\left(Y_{1} \times Y_{2} \times X^{n-2}\right) \leq \mathrm{TC}_{n}(X) .
$$


This leads immediately to the following inequality.

Corollary 2 (See Corollary 2.4) Consider a fibration sequence $F \rightarrow E \rightarrow B$ of connected $C W$ complexes. If the fibration admits a (homotopy) section, then we have

$$
\operatorname{cat}(F \times B) \leq \mathrm{TC}(E) .
$$

We give a number of other applications and consequences of our theorem. For instance, it leads directly to the following inequality due to Dranishnikov [6, Theorem 3.6].

Corollary 3 (See Corollary 2.9) Suppose $X$ and $Y$ have the homotopy type of connected $C W$ complexes. Then $\operatorname{cat}(X \times Y) \leq \mathrm{TC}(X \vee Y)$.

Furthermore, in our actual result we extend this inequality to one involving $\mathrm{TC}_{n}(X \vee Y)$. We also show some results in the rational homotopy setting. The basic result here is of a similar nature to our integral result, but allows for considerably greater flexibility in its application. For our rational results, we assume spaces are simply connected. We refer to Hilton, Mislin and Roitberg [18] for general results about rationalization of simply connected spaces. We write $X_{\mathbb{Q}}$ for the rationalization of a simply connected space $X$, and $g_{\mathbb{Q}}: X_{\mathbb{Q}} \rightarrow Y_{\mathbb{Q}}$ for the rationalization of a map $g: X \rightarrow Y$ of simply connected spaces. Then $\pi_{i}\left(X_{\mathbb{Q}}\right) \cong \pi_{i}(X) \otimes \mathbb{Q}$ are the rational homotopy groups of $X$, and $g_{\mathbb{Q} \#}=g_{\#} \otimes 1: \pi_{*}\left(X_{\mathbb{Q}}\right) \rightarrow \pi_{*}\left(Y_{\mathbb{Q}}\right)$ is the homomorphism induced on rational homotopy groups.

Theorem 4 (See Theorem 3.2) Consider two maps $f_{j}: Y_{j} \rightarrow X$ of simply connected spaces, $j=1,2$, such that each $\left(f_{j \mathbb{Q}}\right)_{\#}: \pi_{*}\left(Y_{j \mathbb{Q}}\right) \rightarrow \pi_{*}\left(X_{\mathbb{Q}}\right)$ is an inclusion on all rational homotopy groups, and the image subgroups satisfy

$$
\left(f_{1 \mathbb{Q}}\right)_{\#}\left(\pi_{i}\left(Y_{1 \mathbb{Q}}\right)\right) \cap\left(f_{2 \mathbb{Q}}\right)_{\#}\left(\pi_{i}\left(Y_{2 \mathbb{Q}}\right)\right)=0 \subseteq \pi_{i}\left(X_{\mathbb{Q}}\right)
$$

for each $i \geq 2$. Then for $n \geq 2$ we have

$$
\operatorname{cat}\left(Y_{1 \mathbb{Q}}\right)+\operatorname{cat}\left(Y_{2 \mathbb{Q}}\right)+(n-2) \operatorname{cat}\left(X_{\mathbb{Q}}\right) \leq \mathrm{TC}_{n}\left(X_{\mathbb{Q}}\right)
$$

The proof we give for Theorem 4 leads to the following consequence.

Corollary 5 (See Corollary 3.3) Let $g: X \rightarrow Z$ and $f: Y \rightarrow Z$ be maps of simply connected spaces. If

(I) $g_{\mathbb{Q} \#:}: \pi_{*}\left(X_{\mathbb{Q}}\right) \rightarrow \pi_{*}\left(Z_{\mathbb{Q}}\right)$ and $f_{\mathbb{Q} \#}: \pi_{*}\left(Y_{\mathbb{Q}}\right) \rightarrow \pi_{*}\left(Z_{\mathbb{Q}}\right)$ are both injective,

(II) $\operatorname{im}\left(g_{\mathbb{Q} \#}\right) \cap \operatorname{im}\left(f_{\mathbb{Q} \#}\right)=0 \subseteq \pi_{*}\left(Z_{\mathbb{Q}}\right)$,

then we have $\operatorname{cat}\left(Y_{\mathbb{Q}}\right) \leq \operatorname{secat}\left(g_{\mathbb{Q}}\right)$. 
Corollary 5 is worth highlighting, as it is a direct generalization of the so-called mapping theorem of Félix and Halperin (see [9, Theorem I] or [12, Theorem 28.6]), a result of fundamental importance in rational homotopy.

Theorem 6 (Félix-Halperin mapping theorem) Let $f: Y \rightarrow Z$ be a map of simply connected spaces. If $f_{\mathbb{Q} \#:} \pi_{*}\left(Y_{\mathbb{Q}}\right) \rightarrow \pi_{*}\left(Z_{\mathbb{Q}}\right)$ is injective, then $\operatorname{cat}\left(Y_{\mathbb{Q}}\right) \leq \operatorname{cat}\left(Z_{\mathbb{Q}}\right)$.

We retrieve the original mapping theorem from Corollary 5 by taking $X$ to be a point; whereupon we have $\operatorname{secat}\left(g_{\mathbb{Q}}\right)=\operatorname{cat}\left(Z_{\mathbb{Q}}\right)$, (I) reduces to the condition that $f_{\mathbb{Q}}$ be injective, (II) becomes vacuous, and the conclusion then $\operatorname{reads} \operatorname{cat}\left(Y_{\mathbb{Q}}\right) \leq \operatorname{cat}\left(Z_{\mathbb{Q}}\right)$. This connection accounts for the title of the paper.

Our rational results also lead to interesting consequences for the global rational homotopy structure of finite complexes. We mention two such here to suggest the kinds of conclusions we are able to draw. For $X$ a simply connected finite complex, we say that $X$ is (rationally) hyperbolic if it has infinitely many non-zero rational homotopy groups. In some sense, this is the generic behavior of a finite complex; see [12, Part VI]. If a simply connected space $X$ has $\mathrm{TC}(X)=2$, then $\mathrm{TC}\left(X_{\mathbb{Q}}\right) \leq 2$, and also cat $\left(X_{\mathbb{Q}}\right) \leq 2$. The interest in the following result lies in the case in which cat $\left(X_{\mathbb{Q}}\right)=2$.

Theorem 7 (See Corollary 5.5) Let $X$ be a simply connected hyperbolic finite complex. If $\mathrm{TC}\left(X_{\mathbb{Q}}\right)=2$, then $X$ has some connective cover that is a rational co-Hspace. In particular, $\pi_{*}(\Omega X) \otimes \mathbb{Q}$ contains a free Lie algebra on two generators.

The Avramov-Félix conjecture [12, Problem 4, Section 39] says that the rational homotopy Lie algebra $\pi_{*}(\Omega X) \otimes \mathbb{Q}$ of a simply connected hyperbolic finite complex should have a sub-Lie algebra that is free on two generators. If true, the conjecture would help explain the phenomenon of exponential growth of rational homotopy groups, about which a great deal of work has been done. The conjecture has been established for $X$ with $\operatorname{cat}\left(X_{\mathbb{Q}}\right)=2$; see Félix, Halperin and Thomas [11; 14]. However, our conclusion here is somewhat stronger and follows by a simple argument, once given our basic result. With our methods, we are able to glean various conclusions in this direction, such as the following new cases of the conjecture.

Corollary 8 (See Corollary 5.8) Let $X$ be a simply connected hyperbolic finite complex with $\operatorname{cat}\left(X_{\mathbb{Q}}\right)=3$ and $\mathrm{TC}_{n}\left(X_{\mathbb{Q}}\right)=3 n-3$ for some $n \geq 2$. Then $\pi_{*}(\Omega X) \otimes \mathbb{Q}$ contains a free Lie algebra on two generators.

Whereas some results of this article concentrate on simply connected spaces, a companion article, Grant, Lupton and Oprea [17], considers analogous results applicable to aspherical spaces. 
The paper is organized as follows. In Section 2 we work in ordinary homotopy theory and establish Theorem 1 of this introduction. Section 3 contains our main rational result which is Theorem 4 of this introduction. In Section 4, we briefly illustrate some situations in which our rational results may be levered to yield calculations of (integral) $\mathrm{TC}(X)$ for certain spaces $X$. In the final Section 5, we apply Theorem 4 to analyze the behavior of $\mathrm{TC}(-)$ with respect to connective covers, and draw our conclusions concerning the Avramov-Félix conjecture.

We finish this introduction with a brief resume of definitions and basic facts. Recall that $\operatorname{cat}(X)$ is the smallest $n$ for which there is an open covering $\left\{U_{0}, \ldots, U_{n}\right\}$ by $n+1$ open sets, each of which is contractible in $X$. Also, for a fibration $p: E \rightarrow B$, $\operatorname{secat}(p)$ is the smallest number $n$ for which there is an open covering $\left\{U_{0}, \ldots, U_{n}\right\}$ of $B$ by $n+1$ open sets, for each of which there is a local section $s_{i}: U_{i} \rightarrow E$ of $p$, so that $p \circ s_{i}=j_{i}: U_{i} \rightarrow B$, where $j_{i}$ denotes the inclusion. Let $P X$ denote the space of (free) paths on a space $X$. There is a fibration $P_{2}: P X \rightarrow X \times X$, which evaluates a path at initial and final points: for $\alpha \in P X$, we have $P_{2}(\alpha)=(\alpha(0), \alpha(1))$. This is a fibrational substitute for the diagonal map $\Delta: X \rightarrow X \times X$. We define $\mathrm{TC}(X):=\operatorname{secat}\left(P_{2}\right)$.

We also consider the "higher analogues" of topological complexity introduced by Rudyak in [22]; see also [23] and Basabe, González, Rudyak and Tamaki [2]. Let $n \geq 2$ and consider the fibration

$$
P_{n}: P X \rightarrow X \times \cdots \times X=X^{n},
$$

defined by

$$
P_{n}(\alpha)=\left(\alpha(0), \alpha\left(\frac{1}{n-1}\right), \ldots, \alpha\left(\frac{n-2}{n-1}\right), \alpha(1)\right)
$$

for $\alpha \in P X$. This is a fibrational substitute for the $n$-fold diagonal $\Delta_{n}: X \rightarrow X^{n}$. Then we define $\mathrm{TC}_{n}(X):=\operatorname{secat}\left(P_{n}\right)$.

Note that the Farber [7] and Rudyak [22] use un-normalized $\mathrm{TC}_{n}(-)$, which is one more than our (normalized) $\mathrm{TC}_{n}(-)$. Most of our results apply to ordinary $\mathrm{TC}(X)$ as well as higher $\operatorname{TC}_{n}(X)$ for $n \geq 3$.

There are several well-known and basic facts which we use throughout the article to compare sectional categories of various maps. We state them here; each is easily justified directly from the definitions. First, suppose given a fibration $p: E \rightarrow B$ and any map $f: B^{\prime} \rightarrow B$. Form the pullback $p^{\prime}: E^{\prime} \rightarrow B^{\prime}$ of $p$ along $f$. Then we have $\operatorname{secat}\left(p^{\prime}\right) \leq \operatorname{secat}(p)$. Next, if $E$ is contractible or, more generally, if $p: E \rightarrow B$ is nullhomotopic, then we have $\operatorname{secat}(p)=\operatorname{cat}(B)$. Combining these ingredients readily 
leads to general inequalities

$$
\operatorname{cat}\left(X^{n-1}\right) \leq \mathrm{TC}_{n}(X) \leq \operatorname{cat}\left(X^{n}\right)
$$

for each $n \geq 2$. These basic facts are explained in Basabe, González, Rudyak and Tamaki [2], and Lupton and Scherer [20], amongst other places.

We frequently use $n$-connective covers of a space. Our notation for this is $X^{[n]}$. Namely, $X^{[n]}$ is the homotopy fibre of the map from $X$ to its $n^{\text {th }}$ Postnikov section, and it is a connected space that satisfies $\pi_{i}\left(X^{[n]}\right)=0$ for $i \leq n$, and $\pi_{i}\left(X^{[n]}\right)=\pi_{i}(X)$ for $i \geq n+1$.

Acknowledgements This work was partially supported by grants from the Simons Foundation (209575 to Gregory Lupton and 244393 to John Oprea).

\section{A lower bound on (higher) topological complexity}

From now on, suppose that spaces $X$ and $Y$ are connected and of the homotopy type of a CW complex. Constructions from them, such as $\Omega X$ or pullbacks that involve them, may be disconnected. We begin with a result that may be well known, but which we cannot find in the literature in the form we need. We include a proof here for completeness. The case $n=2$ is proved in [21, Proposition 3.2]; see also the proof of [5, Theorem 7].

In the following proposition, $\mu: \Omega X \times \Omega X \rightarrow \Omega X$ and $\iota: \Omega X \rightarrow \Omega X$ denote the usual loop multiplication map and the inverse map of loops. Also, $\pi_{j, j+1}=\left(\pi_{j}, \pi_{j+1}\right)$ : $(\Omega X)^{n} \rightarrow(\Omega X) \times(\Omega X)$ for $j=1, \ldots, n-1$ denotes the projection onto the two consecutive $j^{\text {th }}$ and $(j+1)^{\text {st }}$ factors of the product.

Proposition 2.1 For $n \geq 2$, consider the fibration sequence

$$
(\Omega X)^{n-1} \longrightarrow P X \stackrel{P_{n}}{\longrightarrow} X^{n}
$$

of the fibration $P_{n}$ used in the above definition of $\mathrm{TC}_{n}(X)$. Then the connecting map of this fibration sequence may be identified as a map

$$
\partial=\left(\partial_{1}, \ldots, \partial_{n-1}\right): \Omega\left(X^{n}\right)=(\Omega X)^{n} \rightarrow(\Omega X)^{n-1}
$$

with coordinate functions $\partial_{j}=\mu \circ(\iota \times 1) \circ \pi_{j, j+1}$ for each $j=1, \ldots, n-1$. On homotopy groups, we may identify the induced homomorphism $\partial_{\#}: \pi_{r}\left(\Omega X^{n}\right) \rightarrow$ $\pi_{r}\left(\Omega X^{n-1}\right)$ for $r \geq 1$ as a homomorphism of abelian groups

$$
\partial_{\#}: \bigoplus_{j=1}^{n} \pi_{r+1}(X) \rightarrow \bigoplus_{j=1}^{n-1} \pi_{r+1}(X),
$$


where, for $\boldsymbol{a}=\left(a_{1}, \ldots, a_{n}\right) \in \pi_{r}\left(\Omega X^{n}\right)=\bigoplus_{j=1}^{n} \pi_{r+1}(X)$, we have

$$
\partial_{\#}(\boldsymbol{a})=\left(-a_{1}+a_{2},-a_{2}+a_{3}, \ldots,-a_{n-1}+a_{n}\right) .
$$

On sets of components, the induced map of based sets $\partial_{\#}: \pi_{0}\left(\Omega X^{n}\right) \rightarrow \pi_{0}\left(\Omega X^{n-1}\right)$ may be identified as

$$
\partial_{\#}: \prod_{j=1}^{n} \pi_{1}(X) \rightarrow \prod_{j=1}^{n-1} \pi_{1}(X)
$$

with $\partial_{\#}(\boldsymbol{a})=\left(\left(a_{1}\right)^{-1} a_{2},\left(a_{2}\right)^{-1} a_{3}, \ldots,\left(a_{n-1}\right)^{-1} a_{n}\right)$.

Proof We adopt the notation of [26, pages 42-43, 141] for the homotopy fibre of a map. Consider the fibration $P_{n}: P X \rightarrow X^{n}$ as above. The fibre here is $F=(\Omega X)^{n-1}$, and the homotopy fibre is

$$
\boldsymbol{T}^{P_{n}}=\left\{(\alpha, \gamma) \in P X \times \mathcal{P} X^{n} \mid \alpha\left(t_{i}\right)=\gamma_{i+1}(0), i=0, \ldots, n-1\right\},
$$

where $t_{i}=i /(n-1)$, and we have written the coordinate functions of $\gamma$ as $\gamma=$ $\left(\gamma_{1}, \ldots, \gamma_{n}\right): I \rightarrow X^{n}$. A homotopy equivalence $\beta:(\Omega X)^{n-1} \rightarrow \boldsymbol{T}^{P_{n}}$ from fibre to homotopy fibre is given by $\beta(\eta)=\left(\eta, C_{\boldsymbol{x}_{\mathbf{0}}}\right)$, where $C_{x}$ denotes the constant path at $x$, and $\boldsymbol{x}_{\mathbf{0}} \in X^{n}$ denotes the base point $\boldsymbol{x}_{\mathbf{0}}=\left(x_{0}, \ldots, x_{0}\right)$. Now for $\alpha \in P X$, let $\alpha[i]:\left[t_{i-1}, t_{i}\right] \rightarrow X$ denote the restriction of $\alpha$ to the subinterval $\left[t_{i-1}, t_{i}\right]$ for $i=1, \ldots, n-1$. Then the inverse homotopy equivalence may be written explicitly as $\beta^{-1}: \boldsymbol{T}^{P_{n}} \rightarrow(\Omega X)^{n-1}$, with

$$
\beta^{-1}(\alpha, \gamma)=\left(\bar{\gamma}_{1} \alpha[1] \gamma_{2}, \bar{\gamma}_{2} \alpha[2] \gamma_{3}, \ldots, \bar{\gamma}_{n-1} \alpha[n-1] \gamma_{n}\right) .
$$

Here, the notation $\bar{\xi}$ denotes the inverse path to $\xi$, ie $\bar{\xi}(t)=\xi(1-t)$, and juxtaposition of paths denotes their usual composition.

From the pullback of the based path fibration $p_{0}: \mathcal{P} X^{n} \rightarrow X^{n}$ along $P_{n}: P X \rightarrow X^{n}$, we obtain the whisker map $\phi: \Omega X^{n} \rightarrow \boldsymbol{T}^{P_{n}}$ as $\phi(\gamma)=\left(C_{x_{0}}, \gamma\right)$, whence we have the connecting map $\partial: \Omega X^{n} \rightarrow \Omega X^{n-1}$ as

$$
\partial(\gamma)=\beta^{-1} \circ \phi(\gamma)=\left(\bar{\gamma}_{1} \gamma_{2}, \bar{\gamma}_{2} \gamma_{3}, \ldots, \bar{\gamma}_{n-1} \gamma_{n}\right) .
$$

In terms of maps, then, we may identity the $j^{\text {th }}$ component of the connecting map as

$$
\partial=\mu \circ(\iota \times 1) \circ \pi_{j, j+1}: \Omega X^{n} \rightarrow \Omega X \times \Omega X \rightarrow \Omega X \times \Omega X \rightarrow \Omega X
$$

for $j=1, \ldots, n-1$. The assertions about the homomorphisms induced on homotopy groups and sets of components follow, as $\mu$ induces the usual addition of homotopy elements and $\iota$ the inverse (anti-)homomorphism. 
Note that, in the above result, whilst $\pi_{r}\left(\Omega X^{n}\right)$ and $\pi_{r}\left(\Omega X^{n-1}\right)$ are both groups for $r \geq 0$, they may be non-abelian for $r=0$ and so the induced map $\partial_{\#}$ may fail to be a homomorphism for $r=0$.

We will need the following generalization of the direct product of (non-abelian) groups: say that subgroups $A$ and $B$ of a group $G$ are complementary subgroups if they (1) span $G$, that is, if any element of $G$ may be written as a product $a b$ for some $a \in A$ and $b \in B$, and (2) also intersect trivially, that is, if $A \cap B=\{e\}$. Note that if $A$ and $B$ are complementary subgroups of an abelian group $G$, then $G$ is isomorphic to the direct sum $A \oplus B$.

Theorem 2.2 Consider two maps $f_{j}: Y_{j} \rightarrow X$ of connected spaces, $j=1,2$, such that for each $i \geq 1$

(i) each $\left(f_{j}\right)_{\#}: \pi_{i}\left(Y_{j}\right) \rightarrow \pi_{i}(X)$ is an inclusion,

(ii) $\left(f_{1}\right)_{\#}\left(\pi_{i}\left(Y_{1}\right)\right)$ and $\left(f_{2}\right)_{\#}\left(\pi_{i}\left(Y_{2}\right)\right)$ are complementary subgroups in $\pi_{i}(X)$.

Then for $n \geq 2$, we have

$$
\operatorname{cat}\left(Y_{1} \times Y_{2} \times X^{n-2}\right) \leq \mathrm{TC}_{n}(X)
$$

In particular, with $n=2$, we have cat $\left(Y_{1} \times Y_{2}\right) \leq \mathrm{TC}(X)$.

Proof Write $Y=Y_{1} \times Y_{2} \times X^{n-2}$ and $f=f_{1} \times f_{2} \times 1_{X^{n-2}}: Y \rightarrow X^{n}$. Then let $N\left(f, P_{n}\right)$ denote the pullback of $P_{n}$ along $f$. Notice that, since $P_{n}$ is a fibration, this pullback is also a homotopy pullback. Extend this commutative diagram to a homotopy commutative ladder of the fibre sequences of each vertical map, thus

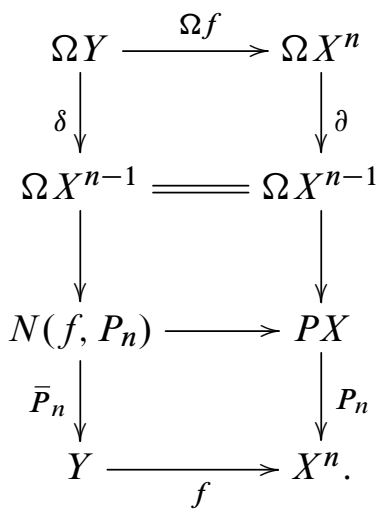

In the long exact homotopy sequence of $\bar{P}_{n}$, identify the connecting map $\delta: \Omega Y \rightarrow$ $\Omega X^{n-1}$ as $\delta=\partial \circ \Omega f$, where $\partial$ denotes the connecting map from Proposition 2.1. So on homotopy groups (or homotopy sets, for $r=0$ ), we may identify the induced map $\delta_{\#}: \pi_{r}(\Omega Y) \rightarrow \pi_{r}\left(\Omega X^{n-1}\right)$ for each $r \geq 0$ as a map 


$$
\delta_{\#}=\partial_{\#} \circ f_{\#}: \pi_{r+1}\left(Y_{1}\right) \times \pi_{r+1}\left(Y_{2}\right) \times \prod_{j=3}^{n} \pi_{r+1}(X) \rightarrow \prod_{j=1}^{n-1} \pi_{r+1}(X) .
$$

With $b_{j} \in \pi_{r+1}\left(Y_{j}\right), j=1,2$, and $a_{j} \in \pi_{r+1}(X), j=3, \ldots, n$, we have

$$
\begin{aligned}
& \delta_{\#}\left(b_{1}, b_{2}, a_{3}, \ldots, a_{n}\right)= \\
& \begin{cases}\left(-f_{1 \#}\left(b_{1}\right)+f_{2 \#}\left(b_{2}\right),-f_{2 \#}\left(b_{2}\right)+a_{3},-a_{3}+a_{4}, \ldots,-a_{n-1}+a_{n}\right) & \text { if } r \geq 1, \\
\left(\left(f_{1 \#}\left(b_{1}\right)\right)^{-1} f_{2 \#}\left(b_{2}\right),\left(f_{2 \#}\left(b_{2}\right)\right)^{-1} a_{3}, a_{3}^{-1} a_{4}, \ldots,\left(a_{n-1}\right)^{-1} a_{n}\right) & \text { if } r=0 .\end{cases}
\end{aligned}
$$

Because the images $f_{1 \#}\left(\pi_{r+1}\left(Y_{1}\right)\right)$ and $f_{2 \#}\left(\pi_{r+1}\left(Y_{2}\right)\right)$ span $\pi_{r+1}(X)$ for each $r \geq 0$, $\delta_{\#}$ is surjective. Because these images intersect only in 0 (or $\{e\}$, in the case $r=0$ ), and because we also assume that $f_{1 \#}$ and $f_{2 \#}$ are injective, it follows that $\delta_{\#}$ is injective. Thus $\delta_{\#}: \pi_{r}(\Omega Y) \rightarrow \pi_{r}\left(\Omega X^{n-1}\right)$ is an isomorphism for each $r \geq 1$, and a bijection of based sets for $r=0$. Therefore $N\left(f, P_{n}\right)$ is weakly contractible, and thus contractible, since it is of the homotopy type of a CW complex.

In the first pullback diagram, then, it follows that $\operatorname{secat}\left(\bar{P}_{n}\right)=\operatorname{cat}(Y)$. Since this diagram is a pullback, we have

$$
\operatorname{cat}(Y)=\operatorname{secat}\left(\bar{P}_{n}\right) \leq \operatorname{secat}\left(P_{n}\right)=\mathrm{TC}_{n}(X) .
$$

Corollary 2.3 For maps $f_{j}: Y_{j} \rightarrow X$ that satisfy the hypotheses of Theorem 2.2, we have $\operatorname{cat}\left(Y_{1}\right) \leq \operatorname{secat}\left(f_{2}\right)$.

Proof Consider the (standard) homotopy pullback

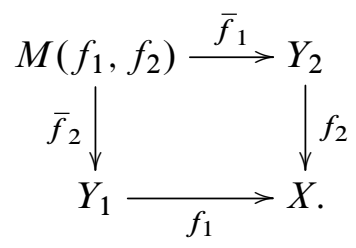

That is,

$$
M\left(f_{1}, f_{2}\right)=\left\{\left(y_{1}, \alpha, y_{2}\right) \in Y_{1} \times \operatorname{map}(I, X) \times Y_{2} \mid \alpha(0)=f_{1}\left(y_{1}\right), \alpha(1)=f_{2}\left(y_{2}\right)\right\} .
$$

Notice that $M\left(f_{1}, f_{2}\right)=N\left(f, P_{2}\right)$, where $f=f_{1} \times f_{2}: Y_{1} \times Y_{2} \rightarrow X \times X$ and $P_{2}: P X \rightarrow X \times X$ are the maps involved in the proof of Theorem 2.2 for $n=2$, and $N\left(f, P_{2}\right)$ is the pullback. Under the hypotheses of Theorem 2.2, we showed that $N\left(f, P_{2}\right)$ is contractible. It follows from the homotopy pullback that $\operatorname{cat}\left(Y_{1}\right)=$ $\operatorname{secat}\left(\bar{f}_{2}\right) \leq \operatorname{secat}\left(f_{2}\right)$.

There is a natural way in which the maps involved in Theorem 2.2 arise. 
Corollary 2.4 Consider a fibration sequence $F \rightarrow E \rightarrow B$ of connected $C W$ complexes. If the fibration admits a (homotopy) section, then for each $n \geq 2$, we have

$$
\operatorname{cat}\left(F \times B \times E^{n-2}\right) \leq \mathrm{TC}_{n}(E) .
$$

Proof The maps $F \rightarrow E$ and $B \rightarrow E$, with the latter being the section, satisfy the conditions of the theorem, with $F, B, E$ identified with $Y_{1}, Y_{2}, X$, respectively.

We present several examples of situations covered by Corollary 2.4. Here, we use $\widetilde{X}$ to denote the universal cover of $X$.

Corollary 2.5 If $X$ has fundamental group $\mathbb{Z}$, then $\operatorname{TC}(X) \geq \operatorname{cat}\left(\widetilde{X} \times S^{1}\right)$. If $X$ has fundamental group $\mathbb{Z} \times \mathbb{Z}$, then $\operatorname{TC}(X) \geq \operatorname{cat}\left(\widetilde{X} \times S^{1} \times S^{1}\right)$.

Proof We argue for $\pi_{1}(X) \cong \mathbb{Z} \times \mathbb{Z}$; the argument for $\pi_{1}(X) \cong \mathbb{Z}$ is identical. Let $k: X \rightarrow S^{1} \times S^{1}=K\left(\pi_{1}(X), 1\right)$ be the classifying map of the universal cover, so that we have a fibre sequence $\widetilde{X} \rightarrow X \rightarrow S^{1} \times S^{1}$. We obtain a section of $k$ by first choosing a map $s: S^{1} \vee S^{1} \rightarrow X$ for which $k \circ s \sim J: S^{1} \vee S^{1} \rightarrow S^{1} \times S^{1}$, where $J: S^{1} \vee S^{1} \rightarrow S^{1} \times S^{1}$ is the inclusion, and noting that $S$ extends to a map $\sigma: S^{1} \times S^{1} \rightarrow X$, as $\pi_{1}(X)$ is abelian. Then $k \circ \sigma: S^{1} \times S^{1} \rightarrow S^{1} \times S^{1}$ induces an isomorphism on $\pi_{1}(-)$ and thus, since $S^{1} \times S^{1}=K\left(\pi_{1}(X), 1\right)$, is a homotopy equivalence. We may adjust $\sigma$, if necessary by pre-composing with a suitable selfhomotopy equivalence of $S^{1} \times S^{1}$, into a section of $k$ up to homotopy. The inequality now follows from Corollary 2.4.

Associated bundles provide another source of applications. Suppose that $p: E \rightarrow M$ is a principal $G$-bundle, for $G$ a topological group. For $X$ a $G$-space, we have the associated bundle $E \times_{G} X \rightarrow M$, with fibre $X$.

Corollary 2.6 With the above notation, if the action of $G$ on the connected space $X$ has a fixed point, then we have TC $\left(E \times{ }_{G} X\right) \geq \operatorname{cat}(X \times M)$.

Proof If the action of $G$ on $X$ has a fixed point, then $E \times_{G} X \rightarrow M$ has a section, and we may apply Corollary 2.4.

Examples 2.7 Here, since the bundle $E \times_{G} X \rightarrow M$ admits a section, we already have the lower bound $\mathrm{TC}\left(E \times_{G} X\right) \geq \mathrm{TC}(M)$. In this situation, therefore, our new lower bound will be useful when $\mathrm{TC}(M)$ is relatively small, compared with cat $(M)$. This happens if, for instance, we also suppose that $M$ is a group, in which case we have $\mathrm{TC}(M)=\operatorname{cat}(M)$; see $[8$, Lemma $8.2 ; 20]$. For example, take the principal circle 
bundle $U(n) \rightarrow P U(n)$, and let $X=S^{2}$ with the rotation action of the circle $S^{1}$. Here we obtain a lower bound of $\operatorname{TC}\left(U(n) \times{ }_{S^{1}} S^{2}\right) \geq \operatorname{cat}\left(S^{2} \times P U(n)\right)$. As another example, take the Hopf bundle $S^{1} \rightarrow S^{3} \rightarrow S^{2}$, and consider any $S^{1}$-action on $\mathbb{C P}^{n}$. The action must have a fixed point, as $\left.\chi(\mathbb{C P})^{n}\right) \neq 0$. We obtain that $\operatorname{TC}\left(S^{3} \times{ }_{S^{1}} \mathbb{C P}^{n}\right) \geq$ $\operatorname{cat}\left(\mathbb{C P}^{n} \times S^{2}\right)=n+1>\operatorname{cat}\left(S^{2}\right)$.

Mapping tori are a further source of applications of Corollary 2.4.

Corollary 2.8 Let $\phi: M \rightarrow M$ be a diffeomorphism of a connected, smooth manifold $M$ and let $M_{\phi}$ denote the corresponding mapping torus. Then $\operatorname{TC}\left(M_{\phi}\right) \geq \operatorname{cat}\left(M \times S^{1}\right)$.

Proof Corollary 2.4 obtains the lower bound, for we have a sectioned fibration $M \rightarrow M_{\phi} \rightarrow S^{1}$.

Using Corollary 2.4, we may also retrieve, and in a very transparent way, (part of) a result of Dranishnikov as well as its extension to higher topological complexity.

Corollary 2.9 Suppose $X$ and $Y$ have the homotopy type of connected CW complexes. Then

$$
\max \left\{\operatorname{cat}\left(X \times Y^{n-1}\right), \operatorname{cat}\left(Y \times X^{n-1}\right)\right\} \leq \operatorname{cat}\left(X \times Y \times(X \vee Y)^{n-2}\right) \leq \mathrm{TC}_{n}(X \vee Y)
$$

for $n \geq 2$. In particular, we have $\operatorname{cat}(X \times Y) \leq \mathrm{TC}(X \vee Y)$.

Proof Let $p_{1}: X \vee Y \rightarrow X$ be projection onto the first summand, $i_{1}: X \rightarrow X \vee Y$ be inclusion into the first summand, and $i_{2}: Y \rightarrow X \vee Y$ be inclusion into the second summand. Because the composition $p_{1} \circ i_{2}: Y \rightarrow X$ is null, there is a lift, up to homotopy, of $i_{2}$ through the (homotopy) fibre inclusion $j: F \rightarrow X \vee Y$. Furthermore, because $i_{2}$ admits the retraction $p_{2}: X \vee Y \rightarrow Y$, this lift admits the retraction $p_{2} \circ j: F \rightarrow Y$. All this is summarized in the following diagram:

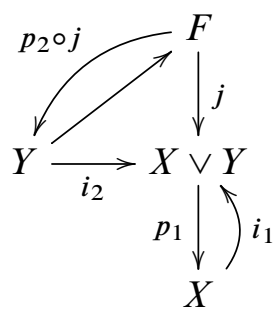

Now Corollary 2.4 gives cat $\left(F \times X \times(X \vee Y)^{n-2}\right) \leq \mathrm{TC}_{n}(X \vee Y)$. But $Y$ is a retract of $F$, and hence $Y \times X \times(X \vee Y)^{n-2}$ is a retract of $F \times X \times(X \vee Y)^{n-2}$. The second inequality follows. The first follows because both $X^{n-2}$ and $Y^{n-2}$ are retracts of $(X \vee Y)^{n-2}$. 


\section{Rational results}

We now turn our attention to the rational homotopy setting. Here, we consider simply connected spaces. Generally, $\mathrm{TC}_{n}\left(X_{\mathbb{Q}}\right)$ provides a lower bound for the (higher) topological complexity. We include a formal statement and proof here for the sake of completeness. We may also consider $P$-localization for this, as we use only generalities about localization. The corresponding inequality for cat(-) is a result of Toomer [25].

Lemma 3.1 Let $f: E \rightarrow B$ be a fibration of simply connected spaces, and let $f_{P}: X_{P} \rightarrow Y_{P}$ be its $P$-localization (at any set of primes $P$ ). Then secat $\left(f_{P}\right) \leq$ $\operatorname{secat}(f)$. In particular, we have $\mathrm{TC}_{n}\left(X_{P}\right)=\operatorname{secat}\left(\left(P_{n}\right)_{P}\right) \leq \operatorname{secat}\left(P_{n}\right)=\mathrm{TC}_{n}(X)$.

Proof We may use general properties of localization (see eg [18, Section II.1]), although not with the covering definition of secat(-) that we have given. Instead, we use Švarc's fibrewise join definition of secat(-). For this, one starts with the pullback along $f$ of $f$, and then forms the pushout of the top left corner to obtain the filler in the following diagram:

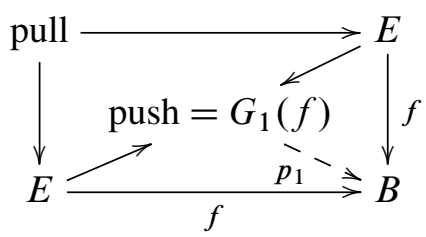

Iterating this pullback-pushout step $n$-times, each time starting with the pullback along $f$ of $p_{i-1}$, results in a fibration $p_{n}: G_{n}(f) \rightarrow Y$, called the $(n+1)$-fold fibrewise join (of $f$ with itself). Under mild conditions (eg $B$ paracompact or normal), a result of Švarc says that secat $(f)$ equals the smallest $n$ for which $p_{n}: G_{n}(f) \rightarrow Y$ has a (global) section. A proof of this identification is given in [15, Theorem 2.2].

Now suppose that $\operatorname{secat}(f)=n$, and that $l_{B}: B \rightarrow B_{P}$ is a $P$-localization map. The functorial nature of the fibrewise join construction results in a commutative diagram

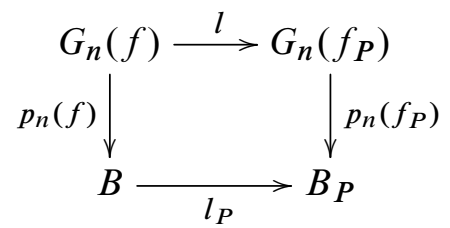

and it is not difficult to identify the right-hand vertical map as $\left(p_{n}\right)_{P}:\left(G_{n}(f)\right)_{P} \rightarrow B_{P}$; see [25] for the case in which $f$ is the path fibration. Since we assume that $\operatorname{secat}(f)=n$, the left-hand vertical map admits a section, and the universal property of the localization map $l_{B}: B \rightarrow B_{P}$ yields a section of $p_{n}\left(f_{P}\right): G_{n}\left(f_{P}\right) \rightarrow B_{P}$. That is, $\operatorname{secat}\left(f_{P}\right) \leq n$. 
The first equality of the last assertion follows from the fact that the induced map $\left(l_{P}\right)_{*}: \operatorname{map}(I, X) \rightarrow \operatorname{map}\left(I, X_{P}\right)$ is a $P$-localization [18, Theorem II.3.11].

Note that the simply connected hypothesis is necessary in Lemma 3.1; the inequality may fail if the spaces are assumed only to be nilpotent. For instance, if we take $X=S^{1}$, then we have $\operatorname{TC}(X)=\operatorname{cat}(X)=1$. However, $X_{\mathbb{Q}}=S_{\mathbb{Q}}^{1}$ is well known to have $\operatorname{cat}\left(S_{\mathbb{Q}}^{1}\right)=2$. Since $S^{1}$ is an H-space, it follows that $X_{\mathbb{Q}}=S_{\mathbb{Q}}^{1}$ is also an $\mathrm{H}$-space. Therefore, we have $\mathrm{TC}\left(X_{\mathbb{Q}}\right)=\operatorname{cat}\left(S_{\mathbb{Q}}^{1}\right)=2$, and $\mathrm{TC}\left(X_{\mathbb{Q}}\right)>\mathrm{TC}(X)$ here.

We begin with a result in this setting whose conclusion is the same as that of Theorem 2.2, but whose hypotheses are weaker (the images of the maps in rational homotopy groups need not span). As we shall see, this allows for greater flexibility in applying the result.

Theorem 3.2 Consider maps $f_{j \mathbb{Q}}: Y_{j \mathbb{Q}} \rightarrow X_{\mathbb{Q}}$ of simply connected rational spaces, $j=1,2$, such that each $\left(f_{j \mathbb{Q}}\right)_{\#}: \pi_{*}\left(Y_{j \mathbb{Q}}\right) \rightarrow \pi_{*}\left(X_{\mathbb{Q}}\right)$ is an inclusion on all (rational) homotopy groups, and the image subgroups satisfy

$$
\left(f_{1 \mathbb{Q}}\right)_{\#}\left(\pi_{i}\left(Y_{1 \mathbb{Q}}\right)\right) \cap\left(f_{2 \mathbb{Q}}\right)_{\#}\left(\pi_{i}\left(Y_{2 \mathbb{Q}}\right)\right)=0 \subseteq \pi_{i}\left(X_{\mathbb{Q}}\right),
$$

for each $i \geq 2$. Then for $n \geq 2$, we have

$$
\operatorname{cat}\left(Y_{1 \mathbb{Q}}\right)+\operatorname{cat}\left(Y_{2 \mathbb{Q}}\right)+(n-2) \operatorname{cat}\left(X_{\mathbb{Q}}\right) \leq \mathrm{TC}_{n}\left(X_{\mathbb{Q}}\right) .
$$

In particular, with $n=2$, we have $\operatorname{cat}\left(Y_{1 \mathbb{Q}}\right)+\operatorname{cat}\left(Y_{2 \mathbb{Q}}\right) \leq \mathrm{TC}\left(X_{\mathbb{Q}}\right)$.

Proof We follow the same steps as in the proof of Theorem 2.2. Write $Y_{\mathbb{Q}}=$

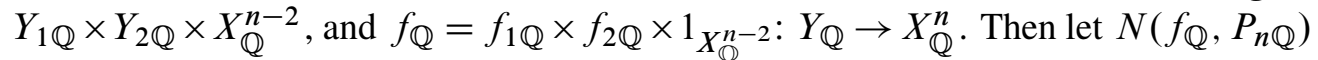
denote the pullback along $f_{\mathbb{Q}}$ of $P_{n \mathbb{Q}}: P X_{\mathbb{Q}} \rightarrow X_{\mathbb{Q}}^{n}$, and construct the same ladder of fibrations as in the proof of Theorem 2.2; thus

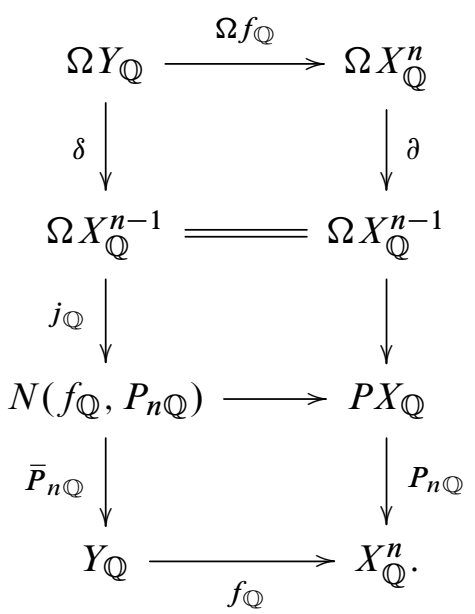


Notice that here we are making various natural identifications, including $\left(X^{n}\right)_{\mathbb{Q}}=$ $\left(X_{\mathbb{Q}}\right)^{n},(\Omega X)_{\mathbb{Q}}=\Omega\left(X_{\mathbb{Q}}\right)$, and $(P X)_{\mathbb{Q}}=P\left(X_{\mathbb{Q}}\right)$. As we did in the proof of Theorem 2.2, in the long exact homotopy sequence of the left-hand vertical map, we now identify the connecting homomorphism (now in rational homotopy groups) $\delta_{\#}: \pi_{i}\left(\Omega Y_{\mathbb{Q}}\right) \rightarrow \pi_{i}\left(\Omega X_{\mathbb{Q}}^{n-1}\right)$ for $i \geq 1$ as an injective homomorphism

$$
\delta_{\#}=\partial_{\#} \circ f_{\mathbb{Q} \#}: \pi_{i+1}\left(Y_{1 \mathbb{Q}}\right) \times \pi_{i+1}\left(Y_{2 \mathbb{Q}}\right) \times \prod_{j=3}^{n} \pi_{i+1}\left(X_{\mathbb{Q}}\right) \rightarrow \prod_{j=1}^{n-1} \pi_{i+1}\left(X_{\mathbb{Q}}\right) .
$$

From the long exact sequence in rational homotopy groups, this implies that the fibre inclusion

$$
j_{\mathbb{Q}}: \Omega X_{\mathbb{Q}}^{n-1} \rightarrow N\left(f_{\mathbb{Q}}, P_{n \mathbb{Q}}\right)
$$

is onto in rational homotopy groups. Now we rely upon a fact peculiar to the rational setting: because the domain of $j_{\mathbb{Q}}$ is a (rational) $\mathrm{H}-$ space, and $j_{\mathbb{Q}}$ is onto in rational homotopy groups, it follows that we have a (rational) section $\sigma: N\left(f_{\mathbb{Q}}, P_{n \mathbb{Q}}\right) \rightarrow$ $\Omega X_{\mathbb{Q}}^{n-1}$ of $j_{\mathbb{Q}}$. Indeed, it is also true that $N\left(f_{\mathbb{Q}}, P_{n \mathbb{Q}}\right)$ is a (rational) H-space; see the proof of the mapping theorem given in [4, Theorem 4.11]. Then it follows that we have $\bar{P}_{n \mathbb{Q}}=\bar{P}_{n \mathbb{Q}} \circ j_{\mathbb{Q}} \circ \sigma=* \circ \sigma=*: N\left(f_{\mathbb{Q}}, P_{n \mathbb{Q}}\right) \rightarrow Y_{\mathbb{Q}}$. Consequently, from the bottom pullback square, we have

$$
\operatorname{cat}\left(Y_{\mathbb{Q}}\right)=\operatorname{secat}\left(\bar{P}_{n \mathbb{Q}}\right) \leq \mathrm{TC}_{n}\left(X_{\mathbb{Q}}\right) .
$$

Finally, we may rewrite $\operatorname{cat}\left(Y_{\mathbb{Q}}\right)$ as the $\operatorname{sum} \operatorname{cat}\left(Y_{1 \mathbb{Q}}\right)+\operatorname{cat}\left(Y_{2 \mathbb{Q}}\right)+(n-2) \operatorname{cat}\left(X_{\mathbb{Q}}\right)$, because the product equality for LS category holds rationally [10].

We may draw the same corollary from this proof as we did from that of Theorem 2.2.

Corollary 3.3 For maps $f_{j \mathbb{Q}}: Y_{j \mathbb{Q}} \rightarrow X_{\mathbb{Q}}$ that satisfy the hypotheses of Theorem 3.2, we have $\operatorname{cat}\left(Y_{1 \mathbb{Q}}\right) \leq \operatorname{secat}\left(f_{2 \mathbb{Q}}\right)$.

Proof Use the argument of Corollary 2.3 with the homotopy pullback

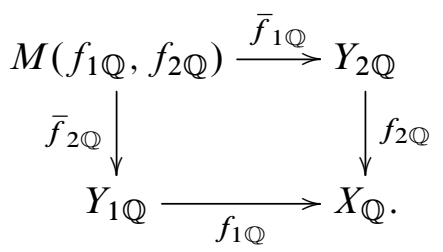

Note that $\bar{f}_{2 \mathbb{Q}}: M\left(f_{1 \mathbb{Q}}, f_{2 \mathbb{Q}}\right) \rightarrow Y_{1 \mathbb{Q}}$ factors through $\bar{P}_{2 \mathbb{Q}}: N\left(f_{\mathbb{Q}}, P_{2 \mathbb{Q}}\right) \rightarrow Y_{\mathbb{Q}}$, where $N\left(f_{\mathbb{Q}}, P_{2 \mathbb{Q}}\right)$ denotes the pullback of $P_{2 \mathbb{Q}}: X_{\mathbb{Q}} \rightarrow X_{\mathbb{Q}} \times X_{\mathbb{Q}}$ along $f_{\mathbb{Q}}=$ $f_{1 \mathbb{Q}} \times f_{2 \mathbb{Q}}: Y_{1 \mathbb{Q}} \times Y_{2 \mathbb{Q}} \rightarrow X_{\mathbb{Q}} \times X_{\mathbb{Q}}$. In the proof of Theorem 3.2, we showed $\bar{P}_{2 \mathbb{Q}}$ to be (rationally) nullhomotopic, and hence $\bar{f}_{2 \mathbb{Q}}$ is nullhomotopic. The result follows. 
We continue with several illustrations of how Theorem 3.2 may be applied. We say that a fibration $p: E \rightarrow B$ of simply connected spaces admits a rational section if the rationalization $p_{\mathbb{Q}}: E_{\mathbb{Q}} \rightarrow B_{\mathbb{Q}}$ admits a section. In this case, we may apply Corollary 2.4 to the rationalized fibration sequence, and conclude that $\operatorname{cat}\left(F_{\mathbb{Q}}\right)+$ $\operatorname{cat}\left(B_{\mathbb{Q}}\right) \leq \mathrm{TC}\left(E_{\mathbb{Q}}\right)$. Because of the relaxed hypotheses of Theorem 3.2, however, we are able to obtain the following somewhat more general result.

Corollary 3.4 Suppose that $i: Y \rightarrow X$ is a map of simply connected spaces that induces an injection on rational homotopy groups, and $F \rightarrow Y \rightarrow B$ is a fibration of $Y$ by simply connected spaces that admits a rational section. Then we have

$$
\operatorname{cat}\left(F_{\mathbb{Q}}\right)+\operatorname{cat}\left(B_{\mathbb{Q}}\right)+(n-2) \operatorname{cat}\left(X_{\mathbb{Q}}\right) \leq \mathrm{TC}_{n}\left(X_{\mathbb{Q}}\right) .
$$

Proof Suppose the maps involved in the fibration of $Y$, after rationalization, are

$$
F_{\mathbb{Q}} \stackrel{j}{\longrightarrow} Y_{\mathbb{Q}} \stackrel{\sigma}{\stackrel{p}{\longrightarrow}} B_{\mathbb{Q}},
$$

with $p \circ \sigma=1: B_{\mathbb{Q}} \rightarrow B_{\mathbb{Q}}$. Then $i \circ j: F_{\mathbb{Q}} \rightarrow X_{\mathbb{Q}}$ and $i \circ \sigma: B_{\mathbb{Q}} \rightarrow X_{\mathbb{Q}}$ satisfy the hypotheses of Theorem 3.2: they both induce injections in rational homotopy groups; and it is easy to see that their image subgroups have trivial intersection in $\pi_{*}\left(X_{\mathbb{Q}}\right)$. Indeed, we have a direct sum decomposition $\pi_{*}\left(Y_{\mathbb{Q}}\right) \cong j_{\#}\left(\pi_{*}\left(F_{\mathbb{Q}}\right)\right) \oplus \sigma_{\#}\left(\pi_{*}\left(B_{\mathbb{Q}}\right)\right)$. Suppose we have $a \in \pi_{*}\left(F_{\mathbb{Q}}\right), b \in \pi_{*}\left(B_{\mathbb{Q}}\right)$ such that $(i \circ j)_{\#}(a)=(i \circ \sigma)_{\#}(b) \in \pi_{*}\left(X_{\mathbb{Q}}\right)$. Then $i_{\#}\left(j_{\#}(a)-\sigma_{\#}(b)\right)=0$. Hence $j_{\#}(a)-\sigma_{\#}(b)=0 \in \pi_{*}\left(Y_{\mathbb{Q}}\right)$, as $i$ is injective in rational homotopy, and thus we have $a=0 \in \pi_{*}\left(F_{\mathbb{Q}}\right)$ and $b=0 \in \pi_{*}\left(B_{\mathbb{Q}}\right)$, since $j_{\#}\left(\pi_{*}\left(F_{\mathbb{Q}}\right)\right) \cap \sigma_{\#}\left(\pi_{*}\left(B_{\mathbb{Q}}\right)\right)=0 \in \pi_{*}\left(Y_{\mathbb{Q}}\right)$, and both $j$ and $\sigma$ are injective in rational homotopy. The result follows.

In the next example, and at several places in the sequel, we make use of minimal models. These are a basic tool of rational homotopy theory that allows one to work in an entirely algebraic setting. We refer to [12, Section 12 et seq] and [13, Chapter 2] for comprehensive treatments of minimal models. Here, we simply recall that the minimal model of a space $X$ is a differential graded (DG) algebra $\bigwedge(V ; d)$, where $\bigwedge V$ denotes the free graded-commutative algebra generated by the graded vector space $V$ and $d$ denotes a decomposable differential, that is, we have $d: V \rightarrow \wedge^{\geq 2} V$. The minimal model encodes all of the rational homotopy information of $X$, in a certain technical sense. Some rational homotopy data are readily extracted from it. For example, on passing to cohomology we obtain $H(\bigwedge(V ; d)) \cong H^{*}(X ; \mathbb{Q})$, and as graded vector spaces we have isomorphisms $\operatorname{Hom}(V, \mathbb{Q}) \cong \pi_{*}(X) \otimes \mathbb{Q}$. As another example, Whitehead product structure in $\pi_{*}(X) \otimes \mathbb{Q}$ corresponds to the quadratic part of the differential $d$; see [12, Section 13(e)] for details. 
A number of authors have used algebraic or minimal model versions of TC( - ) to study rationalized topological complexity; eg [15; 19]. Whilst we use minimal models in some of our examples and applications, we do not make use of an algebraic, or minimal model, version of $\mathrm{TC}_{n}(-)$ as such.

Example 3.5 We analyze an example from [15]. Let $X=S^{3} \vee S^{3} \cup_{a} e^{8} \cup_{b} e^{8}$, with the 8 -cells attached by Whitehead products $a=\left[\iota_{1},\left[\iota_{1}, \iota_{2}\right]\right]$ and $b=\left[\iota_{2},\left[\iota_{1}, \iota_{2}\right]\right]$. In [15, Example 6.5], it is shown that the so-called module topological complexity is $\operatorname{MTC}(X)=3$, which gives a better lower bound for (rational) $\mathrm{TC}(X)$ than does the rational zero-divisors lower bound, which is 2 here. We will use Theorem 3.2 to match this lower bound given by $\operatorname{MTC}(X)$.

We construct maps $f_{j}: Y_{j} \rightarrow X$ with the salient properties. We may describe (the first few terms of) the minimal model of $X$ as

$$
\bigwedge\left(u_{3}, v_{3}, w_{5}, x_{10}, \ldots\right),
$$

with subscripts denoting degrees, and differentials $d(u)=0=d(v), d(w)=u v$, $d(x)=u v w$, and so on. Notice that the cycles $u w$ and $v w$ generate cohomology in degree 8 , and that $X$ is not formal. Now we have an obvious projection of the minimal model

$$
\bigwedge\left(u_{3}, v_{3}, w_{5}, x_{10}, \ldots\right) \rightarrow \bigwedge(u, w ; \bar{d}=0)
$$

that corresponds to a map $f_{1}: Y_{1} \rightarrow X$ injective in rational homotopy. Indeed, $Y_{1} \simeq \mathbb{Q}$ $S^{3} \times S^{5}$. On the other hand, we also have a projection

$$
\bigwedge\left(u_{3}, v_{3}, w_{5}, x_{10}, \ldots\right) \rightarrow \bigwedge(v, x, \ldots) .
$$

Here the quotient differential will be zero for the first terms, but it is generally not zero. This likewise corresponds to a map $f_{2}: Y_{2} \rightarrow X$ injective in rational homotopy. Note that $Y_{2}$ fibres rationally over $S^{3}$ with fibre $X^{[9]}$, the 9-connective cover of $X$. The images $\left(f_{j}\right)_{\#}\left(\pi_{*}\left(Y_{j \mathbb{Q}}\right)\right)$ in $\pi_{*}\left(X_{\mathbb{Q}}\right)$ are distinct. Indeed, the only degree in which they could possibly intersect non-trivially is in degree 3 , and there the images are distinct by choice. Applying Theorem 3.2, we obtain that

$$
\operatorname{TC}\left(X_{\mathbb{Q}}\right) \geq \operatorname{cat}\left(Y_{\mathbb{Q}}\right)=\operatorname{cat}\left(Y_{1 \mathbb{Q}}\right)+\operatorname{cat}\left(Y_{2 \mathbb{Q}}\right) \geq 2+1=3
$$

since $Y_{2}$ is not rationally contractible. In fact we have $\mathrm{TC}\left(X_{\mathbb{Q}}\right) \leq 3$ in this example, as follows from [15, Example 6.5] and [19, Proposition 1.5]. It follows that the space $Y_{2}$ here is a rational co-H-space.

Connective covers provide one source of spaces $Y_{j}$ for Theorem 3.2. Since the map $X^{[N]} \rightarrow X$ is injective in (rational) homotopy groups, the mapping theorem (Theorem 6) 
yields $\operatorname{cat}\left(X_{\mathbb{Q}}^{[N]}\right) \leq \operatorname{cat}\left(X_{\mathbb{Q}}\right)$ for any $N \geq 2$. We illustrate the use of connective covers in this setting with a proof of a rational version of the main result of [16].

Corollary 3.6 Let $X$ be a simply connected space with $\mathrm{TC}_{n}\left(X_{\mathbb{Q}}\right)=n-1$ for some $n \geq 2$. Then $X \simeq_{\mathbb{Q}} S^{2 r+1}$ for some $r \geq 1$.

Proof First, $X$ must have at least one non-zero odd-degree rational homotopy group. Otherwise $X$ would be rationally equivalent to a product of even-dimensional EilenbergMac Lane spaces, and we would have $\infty=\operatorname{cat}\left(X_{\mathbb{Q}}\right)=\mathrm{TC}\left(X_{\mathbb{Q}}\right)$. So suppose that $\pi_{2 r+1}\left(X_{\mathbb{Q}}\right) \neq 0$, for some $r \geq 1$, and that all odd-degree rational homotopy groups of $X$ below this degree are zero. Then we may fibre the minimal model of $X^{[2 r]}$ as

$$
\bigwedge(v) \longleftrightarrow(\bigwedge(v) \otimes \wedge(W), d) \longrightarrow(\bigwedge(W), \bar{d}),
$$

with $v$ a generator in degree $2 r+1$. Topologically, this corresponds to a fibration with section

$$
F_{\mathbb{Q}} \stackrel{j}{\longrightarrow} X_{\mathbb{Q}}^{[2 r]} \stackrel{\stackrel{\sigma}{\rightleftarrows}}{\stackrel{p}{\longrightarrow}} S_{\mathbb{Q}}^{2 r+1} .
$$

Note that, since $(n-1) \operatorname{cat}\left(X_{\mathbb{Q}}\right) \leq \mathrm{TC}_{n}\left(X_{\mathbb{Q}}\right)$, our hypothesis implies that cat $\left(X_{\mathbb{Q}}\right)=1$, so that $X$ is a rational co-H-space. Note also that we have $\operatorname{cat}\left(S_{\mathbb{Q}}^{2 r+1}\right)=1$. Now apply Corollary 3.4, and obtain that $\operatorname{cat}\left(F_{\mathbb{Q}}\right)+1+(n-2) \leq \mathrm{TC}\left(X_{\mathbb{Q}}\right)=n-1$, whence $\operatorname{cat}\left(F_{\mathbb{Q}}\right)=0$, and so $X^{[2 r]} \simeq \mathbb{Q} S^{2 r+1}$.

Two possibilities remain. Either we have all rational homotopy groups of degree below $2 r+1$ zero, in which case $X \simeq \mathbb{Q} S^{2 r+1}$. Or, if there are non-zero rational homotopy groups of degree less than $2 r+1$, the minimal model of $X$ must be of the form $\bigwedge\left(u_{1}, \ldots, u_{k}, v ; d\right)$, with each $u_{i}$ of even degree. In this latter case, the only possible non-zero differential is $d(v)=P$, where $P$ is some polynomial in degree $2 r+2$ in the $u_{i}$. A straightforward argument shows that if $k \geq 2$, then $X_{\mathbb{Q}}$ has a non-zero cup product in cohomology — indeed, must have infinite cup length — and hence $X$ cannot be a rational co-H-space. On the other hand, if $k=1$, then $H^{*}(X ; \mathbb{Q}) \cong \mathbb{Q}\left[u_{1}\right] /\left(u_{1}^{n}\right)$ is a truncated polynomial algebra on a single even-degree generator. For such a space, the zero-divisors lower bound implies that $\mathrm{TC}\left(X_{\mathbb{Q}}\right) \geq 2$, and so this case may also be eliminated. The only remaining possibility, then, is that $X \simeq_{\mathbb{Q}} S^{2 r+1}$.

We give another consequence of Corollary 3.4 of a rather general nature.

Corollary 3.7 Let $X$ be a simply connected hyperbolic space. Suppose that we have linearly independent elements $a \in \pi_{2 r+1}\left(X_{\mathbb{Q}}\right)$ and $b \in \pi_{2 s+1}\left(X_{\mathbb{Q}}\right), r \leq s$, with zero Whitehead product: $[a, b]=0 \in \pi_{2 r+2 s+1}\left(X_{\mathbb{Q}}\right)$. Then we have $\operatorname{TC}_{n}\left(X_{\mathbb{Q}}\right) \geq 2 n-1$ for each $n \geq 2$. 
Proof We argue with minimal models. First, write the minimal model of $X$ as

$$
\bigwedge\left(x_{1}, \ldots, x_{k}, a, x_{k+1}, \ldots, x_{l}, b, w_{1}, \ldots ; d\right)
$$

with generators in non-decreasing degree order and, by abuse of notation, with $a$ and $b$ the odd-degree generators that correspond to the linearly independent rational homotopy elements in the hypotheses. Then the ideal generated by $\left\{x_{1}, \ldots, x_{k}, x_{k+1}, \ldots, x_{l}\right\}$ (without $a$ ) is $d$-stable, and we may project onto the quotient by this ideal:

$$
\bigwedge\left(x_{1}, \ldots, x_{k}, a, x_{k+1}, \ldots, x_{l}, b, w_{1}, \ldots ; d\right) \rightarrow \bigwedge\left(a, b, w_{1}, \ldots ; \bar{d}\right) .
$$

This corresponds to a map $Y_{\mathbb{Q}} \rightarrow X_{\mathbb{Q}}$ that is injective in rational homotopy groups. Next we may fibre the model $\bigwedge\left(a, b, w_{1}, \ldots ; \bar{d}\right)$ of $Y$ as

$$
\bigwedge(a, b) \longleftrightarrow(\bigwedge(a, b) \otimes \wedge(W), \bar{d}) \longrightarrow\left(\bigwedge(W), \bar{d}^{\prime}\right)
$$

which corresponds to a fibration with section

$$
F_{\mathbb{Q}} \stackrel{j}{\longrightarrow} Y_{\mathbb{Q}} \underset{p}{\stackrel{\sigma}{\longrightarrow} S_{\mathbb{Q}}^{2 r+1}} \times S_{\mathbb{Q}}^{2 s+1} .
$$

Note that we obtain the section (retraction in minimal models) because of the assumption about the Whitehead product vanishing: in minimal models, this means that the term $a b$ does not occur in any differential (in $d$, and hence in $\bar{d}^{\prime}$ ). Because we assume that $X$ is hyperbolic, it follows that $Y$ is hyperbolic and, in particular, $F_{\mathbb{Q}}$ cannot be contractible. Now apply Corollary 3.4 to obtain that $\mathrm{TC}_{n}\left(X_{\mathbb{Q}}\right) \geq \operatorname{cat}\left(F_{\mathbb{Q}}\right)+2+(n-2) \operatorname{cat}\left(X_{\mathbb{Q}}\right)$. Since $X_{\mathbb{Q}}$ does not have a free homotopy Lie algebra, we have that $\operatorname{cat}\left(X_{\mathbb{Q}}\right) \geq 2$. Hence we have $\mathrm{TC}_{n}\left(X_{\mathbb{Q}}\right) \geq \operatorname{cat}\left(F_{\mathbb{Q}}\right)+2 n-2 \geq 2 n-1$, since $\operatorname{cat}\left(F_{\mathbb{Q}}\right) \geq 1$.

This last result indicates an intriguing connection between (the vanishing of) products in the homotopy Lie algebra and TC(-), which we pursue in more detail in the last section.

\section{From rational results to integral results}

In this section, we illustrate how our rational lower bounds may lead to a determination of ordinary (higher) TC(-). Let $X$ be a simply connected space of finite type. We say that $X$ is an oddly generated space if its rational homotopy groups are concentrated in finitely many odd degrees. In this case, $X$ has minimal model of the form $\bigwedge\left(v_{1}, \ldots, v_{k} ; d\right)$, with each $\left|v_{i}\right|$ odd, not necessarily of distinct degrees, and then we say that $X$ has rank $k$. Any iterated sequence of principal bundles starting from a product of odd spheres has minimal model of this form. Lie groups themselves have such models with zero differential. 
Theorem 4.1 Suppose $X$ and $Y$ are simply connected oddly generated spaces, both of rank $k$. If $X$ and $Y$ satisfy $\operatorname{cat}(X)=\operatorname{cat}(Y)=k$, then $\mathrm{TC}_{n}(X \vee Y)=n k$.

Proof For any oddly generated space $X$ of rank $k$, we have cat $\left(X_{\mathbb{Q}}\right)=k$. Applying the rational version of Corollary 2.9,

$$
n k=\operatorname{cat}\left(X_{\mathbb{Q}}\right)+\operatorname{cat}\left(Y_{\mathbb{Q}}\right)+(n-2) \max \left\{\operatorname{cat}\left(X_{\mathbb{Q}}\right), \operatorname{cat}\left(Y_{\mathbb{Q}}\right)\right\} \leq \mathrm{TC}_{n}\left(X_{\mathbb{Q}} \vee Y_{\mathbb{Q}}\right) .
$$

Now $\mathrm{TC}_{n}\left(X_{\mathbb{Q}} \vee Y_{\mathbb{Q}}\right) \leq \mathrm{TC}_{n}(X \vee Y)$, and we have a general upper bound of

$$
\mathrm{TC}_{n}(X \vee Y) \leq \operatorname{cat}\left((X \vee Y)^{n}\right) \leq n \max \{\operatorname{cat}(X), \operatorname{cat}(Y)\} .
$$

Under our hypotheses, the result follows.

For instance, we may take $X$ or $Y$ to be one of: a product of $k$ odd-dimensional spheres, $U(k)$, or $S U(k+1)$; see [24] or [4, Proposition 9.5, Theorem 9.47]. It is not too hard to construct further examples of oddly generated spaces to which Theorem 4.1 may be applied, resulting in an exact determination of ordinary, and not just rational, $\mathrm{TC}_{n}(-)$.

Example 4.2 Here we construct a smooth manifold $X$ that is oddly generated with $\operatorname{rank}(X)=\operatorname{cat}(X)=3$. Take $p: E \rightarrow S^{6}$ to be the (unit) sphere bundle of the tangent bundle over $S^{6}$. This is an $S^{5}$-bundle over $S^{6}$. Now let $f: S^{3} \times S^{3} \rightarrow S^{6}$ be a (smooth) map of degree 1, and form the pullback

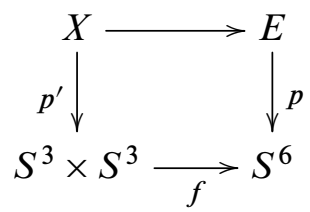

of $p$ along $f$. This results in our space $X$, an $S^{5}$-bundle over $S^{3} \times S^{3}$. We see that the minimal model of $X$ is $\bigwedge\left(v_{1}, v_{2}, v_{3} ; d\right)$, with $\left|v_{1}\right|=\left|v_{2}\right|=3$ and $\left|v_{3}\right|=5$, with differential given by $d\left(v_{3}\right)=v_{1} v_{2}$ (this kind of example is discussed in [13]). Using rational category (in fact, the rational Toomer invariant) as a lower bound, and the usual "dimension over connectivity" upper bound [4, Theorem 1.49], we find that

$$
3=\mathrm{e}_{0}(X) \leq \operatorname{cat}\left(X_{\mathbb{Q}}\right) \leq \operatorname{cat}(X) \leq \frac{\operatorname{dim}(X)}{3}=\frac{11}{3}<4,
$$

and thus we have $\operatorname{cat}(X)=3$. Then we have, for example, $\operatorname{TC}(X \vee X)=6=$ $\operatorname{cat}((X \vee X) \times(X \vee X))$ from Theorem 4.1. 


\section{Consequences for the Avramov-Félix conjecture}

Conjecture 5.1 (Avramov-Félix) If $X$ is a simply connected hyperbolic finite complex, then $\pi_{*}(\Omega X) \otimes \mathbb{Q}$ contains a free Lie algebra on two generators.

This has been established in some cases, including the case in which $X$ has $\operatorname{cat}\left(X_{\mathbb{Q}}\right)=2$ (see [11], although some sub-cases are left open), the case in which $\pi_{*}(\Omega X) \otimes \mathbb{Q}$ has depth 1 (see [3], which covers the sub-cases left open for $\operatorname{cat}\left(X_{\mathbb{Q}}\right)=2$ ), and the case in which $X$ is a Poincaré duality space, with $H^{*}(X ; \mathbb{Q})$ evenly graded and generated by at most three generators (implied by the results of [1]).

For a given value of $\operatorname{cat}\left(X_{\mathbb{Q}}\right)$, the general inequalities $\operatorname{cat}\left(X^{n-1}\right) \leq \mathrm{TC}_{n}(X) \leq \operatorname{cat}\left(X^{n}\right)$ for each $n \geq 2$, imply that $\mathrm{TC}_{n}\left(X_{\mathbb{Q}}\right)$ is lowest when $\mathrm{TC}_{n}\left(X_{\mathbb{Q}}\right)=(n-1) \operatorname{cat}\left(X_{\mathbb{Q}}\right)$. Said differently, amongst spaces with $\operatorname{cat}\left(X_{\mathbb{Q}}\right)=k$, those with $\mathrm{TC}_{n}\left(X_{\mathbb{Q}}\right)=(n-1) k$ for some $n \geq 2$ satisfy a particularly strong constraint, such that one might hope to prove theorems about them. An extreme case of this point of view is given in Corollary 3.6, and its integral counterpart in [16]. Here we make some progress on the Avramov-Félix $(\mathrm{AF})$ conjecture for spaces that satisfy $\mathrm{TC}_{n}\left(X_{\mathbb{Q}}\right)=(n-1) \operatorname{cat}\left(X_{\mathbb{Q}}\right)$.

A rational co-H-space, that is, a simply connected space $X$ with $\operatorname{cat}\left(X_{\mathbb{Q}}\right)=1$, has rational homotopy Lie algebra $\pi_{*}(\Omega X) \otimes \mathbb{Q}$, a free graded Lie algebra, and thus automatically satisfies the AF conjecture, assuming it is rationally hyperbolic. Also, for any connective cover $X^{[N]}$ of a simply connected, finite-type space $X$, we have an inclusion of rational homotopy Lie algebras $\pi_{*}\left(\Omega X^{[N]}\right) \otimes \mathbb{Q} \subseteq \pi_{*}(\Omega X) \otimes \mathbb{Q}$. Furthermore, by Theorem 6 , we know that $\operatorname{cat}\left(X_{\mathbb{Q}}^{[N]}\right) \leq \operatorname{cat}\left(X_{\mathbb{Q}}\right)$ for any cover, however there is no guarantee that cat(-) will actually decrease upon passing to a cover. Our basic strategy here is to consider connective covers, attempting to reduce $\operatorname{cat}\left(X_{\mathbb{Q}}^{[N]}\right)$ to a value for which the AF conjecture is known to hold.

This strategy prompts the following questions.

Question 5.2 When does a simply connected hyperbolic finite complex have a connective cover that is a rational co-H-space? More generally, when does a simply connected hyperbolic finite complex have a connective cover of strictly lower rational category?

Example 5.3 We observe that not every space has a connective cover that is a rational co-H-space, and not every space has a connective cover of strictly lower rational category: Take $X=\left(S^{3} \vee S^{3}\right) \times\left(S^{3} \vee S^{3}\right)$. For each $N$, we have

$$
X^{[N]}=\left(S^{3} \vee S^{3}\right)^{[N]} \times\left(S^{3} \vee S^{3}\right)^{[N]} .
$$


It is easy to see here that $\operatorname{cat}\left(X_{\mathbb{Q}}\right)=\operatorname{cat}\left(X_{\mathbb{Q}}^{[N]}\right)=2$ for each $N$. We may adapt this example to one in which $\operatorname{cat}\left(X_{\mathbb{Q}}^{[N]}\right)$ decreases from $\operatorname{cat}\left(X_{\mathbb{Q}}\right)$ to any intermediate value between $\operatorname{cat}\left(X_{\mathbb{Q}}\right)$ and 1 , and stabilizes at that value. For instance, consider $X=\left(S^{3} \vee S^{3}\right) \times\left(S^{3} \vee S^{3}\right) \times S^{5} \times S^{7}$. Then $\operatorname{cat}\left(X_{\mathbb{Q}}\right)=4$, cat $\left(X_{\mathbb{Q}}^{[5]}\right)=3$, and $\operatorname{cat}\left(X_{\mathbb{Q}}^{[N]}\right)=2$ for $N \geq 7$.

We give a partial response to Question 5.2 in our next result. We will then specialize to small values of $\operatorname{cat}\left(X_{\mathbb{Q}}\right)(\leq 3)$ and establish some cases of the AF conjecture.

In the following results, note that $\pi_{\text {odd }}(X) \otimes \mathbb{Q}$ must be non-zero, otherwise $X$ would have the rational homotopy type of a product of even-dimensional Eilenberg-Mac Lane spaces, and could not be finite.

Theorem 5.4 Let $X$ be a simply connected hyperbolic finite complex. Suppose that we have $\mathrm{TC}_{n}\left(X_{\mathbb{Q}}\right)=(n-1) \operatorname{cat}\left(X_{\mathbb{Q}}\right)$ for some $n \geq 2$.

(1) Suppose $2 r+1$ is the lowest odd degree in which $\pi_{*}(X) \otimes \mathbb{Q}$ is non-zero. Then

$$
\operatorname{cat}\left(X_{\mathbb{Q}}^{[2 r+1]}\right) \leq \operatorname{cat}\left(X_{\mathbb{Q}}\right)-1 .
$$

(2) Suppose that there exist linearly independent elements $a \in \pi_{2 p}(\Omega X) \otimes \mathbb{Q}, b \in$ $\pi_{2 p}(\Omega X) \otimes \mathbb{Q}, p \leq q$, with Samelson product $\langle a, b\rangle=0 \in \pi_{2 p+2 q}(\Omega X) \otimes \mathbb{Q}$. Then

$$
\operatorname{cat}\left(X_{\mathbb{Q}}^{[2 q+1]}\right) \leq \operatorname{cat}\left(X_{\mathbb{Q}}\right)-2
$$

Proof (1) Choose any essential map $\alpha: S^{2 r+1} \rightarrow X_{\mathbb{Q}}$. Because odd-dimensional spheres are rational Eilenberg-Mac Lane spaces, $\alpha$ is injective in (all) rational homotopy groups. Then the maps $\alpha: Y_{1}=S^{2 r+1} \rightarrow X_{\mathbb{Q}}$ and $i: X_{\mathbb{Q}}^{[2 r+1]} \rightarrow X_{\mathbb{Q}}$ satisfy the hypotheses of Theorem 3.2, yielding an inequality

$$
1+\operatorname{cat}\left(X_{\mathbb{Q}}^{[2 r+1]}\right)+(n-2) \operatorname{cat}\left(X_{\mathbb{Q}}\right) \leq \mathrm{TC}_{n}\left(X_{\mathbb{Q}}\right)=(n-1) \operatorname{cat}\left(X_{\mathbb{Q}}\right) .
$$

It follows that we must have $\operatorname{cat}\left(X_{\mathbb{Q}}^{[2 r+1]}\right) \leq \operatorname{cat}\left(X_{\mathbb{Q}}\right)-1$, as asserted.

(2) Under the standard identifications of $\pi_{*}(\Omega X)$ with $\pi_{*+1}(X)$, and Samelson product in $\pi_{*}(\Omega X)$ with Whitehead product in $\pi_{*}(X)$, the assumption may be phrased as follows: For linearly independent elements $\alpha \in \pi_{2 p+1}\left(X_{\mathbb{Q}}\right), \beta \in \pi_{2 q+1}\left(X_{\mathbb{Q}}\right)$, we have $[\alpha, \beta]=0 \in \pi_{2 p+2 q+1}\left(X_{\mathbb{Q}}\right)$, where the bracket denotes their Whitehead product. Because their Whitehead product is zero, we have a map $f_{1}: Y_{1}=S^{2 p+1} \times S^{2 q+1} \rightarrow X_{\mathbb{Q}}$ that restricts to $(\alpha \mid \beta): S^{2 p+1} \vee S^{2 q+1} \rightarrow X_{\mathbb{Q}}$ on the wedge. Once again, because odd-dimensional spheres are rational Eilenberg-Mac Lane spaces, $f_{1}$ is injective in 
(all) rational homotopy groups. Then the maps $f_{1}$ and $f_{2}: Y_{2}=X_{\mathbb{Q}}^{[2 q+1]} \rightarrow X_{\mathbb{Q}}$ satisfy the hypotheses of Theorem 3.2, and we obtain an inequality

$$
2+\operatorname{cat}\left(X_{\mathbb{Q}}^{[2 q+1]}\right)+(n-2) \operatorname{cat}\left(X_{\mathbb{Q}}\right) \leq \mathrm{TC}_{n}\left(X_{\mathbb{Q}}\right)=(n-1) \operatorname{cat}\left(X_{\mathbb{Q}}\right),
$$

whence $\operatorname{cat}\left(X_{\mathbb{Q}}^{[2 q+1]}\right) \leq \operatorname{cat}\left(X_{\mathbb{Q}}\right)-2$.

For a graded Lie algebra $L$, we say that $L$ has no zero brackets if, whenever $x, y \in L$ are linearly independent elements, we have $[x, y] \neq 0 \in L$.

Recall again the general inequality $(n-1) \operatorname{cat}\left(X_{\mathbb{Q}}\right) \leq \mathrm{TC}_{n}\left(X_{\mathbb{Q}}\right)$, for each $n \geq 2$. If we assume that $\mathrm{TC}_{n}\left(X_{\mathbb{Q}}\right) \leq 2 n-3$ for some $n$, then it follows that $\operatorname{cat}\left(X_{\mathbb{Q}}\right)=1$, and $X$ is a rational co-H-space. Since we are assuming $X$ is hyperbolic, this implies that $X$ has the same rational homotopy type as a wedge of at least two spheres, and hence satisfies the AF conjecture. The next step, therefore, is the case in which $\mathrm{TC}_{n}\left(X_{\mathbb{Q}}\right) \leq 2 n-2$ for some $n \geq 2$. The interest here is in the case in which cat $\left(X_{\mathbb{Q}}\right)=2$ and $\mathrm{TC}_{n}\left(X_{\mathbb{Q}}\right)=2 n-2$ for some $n$. Any space with cat $\left(X_{\mathbb{Q}}\right)=2$ is already known to satisfy the AF conjecture $[11 ; 3]$. Here, we will obtain a stronger conclusion using the extra constraint on $\mathrm{TC}_{n}\left(X_{\mathbb{Q}}\right)$.

Corollary 5.5 Let $X$ be a simply connected hyperbolic finite complex, and suppose $\mathrm{TC}_{n}\left(X_{\mathbb{Q}}\right) \leq 2 n-2$ for some $n \geq 2$. Then $\pi_{\text {even }}(\Omega X) \otimes \mathbb{Q}$ has no zero brackets. Furthermore, if $\pi_{2 \mathrm{r}}(\Omega X) \otimes \mathbb{Q}$ is the lowest-degree non-zero part of $\pi_{\mathrm{even}}(\Omega X) \otimes \mathbb{Q}$, then the connective cover $X_{\mathbb{Q}}^{[2 r+1]}$ is a (rational) co-H-space. In particular, $\pi_{*}(\Omega X) \otimes \mathbb{Q}$ contains as a sub-Lie algebra the infinite-dimensional free Lie algebra $\pi_{*}\left(\Omega X^{[2 r+1]}\right) \otimes \mathbb{Q}$.

Proof The hypothesis $\mathrm{TC}_{n}\left(X_{\mathbb{Q}}\right) \leq 2 n-2$ for some $n \geq 2$ entails cat $\left(X_{\mathbb{Q}}\right) \leq 2$. If $\operatorname{cat}\left(X_{\mathbb{Q}}\right)=1$, then $\pi_{*}(\Omega X) \otimes \mathbb{Q}$ is a free Lie algebra and we are done. So assume that $\operatorname{cat}\left(X_{\mathbb{Q}}\right)=2$. First, suppose that we have two linearly independent, even-degree elements in $\pi_{*}(\Omega X) \otimes \mathbb{Q}$ whose bracket is zero. Then part (2) of Theorem 5.4 contradicts the fact that each connective cover $X_{\mathbb{Q}}^{[N]}$ is non-contractible. Hence, $\pi_{\text {even }}(\Omega X) \otimes \mathbb{Q}$ has no zero brackets. The remaining assertion follows directly from part (1) of Theorem 5.4.

Remark 5.6 The condition that a graded Lie algebra have no zero brackets is, in general, strictly weaker than the condition that the Lie algebra be free. If a graded Lie algebra is free, then it has no zero brackets. The example below illustrates that the converse need not be true. Note, however, that our example is not the rational homotopy Lie algebra of a hyperbolic space, as it does not have the (exponentially) increasing ranks displayed by such. Clearly, no zero brackets entails a rich bracket structure. It would be interesting to understand more fully the relationship, if any, between no zero brackets and freeness in the context of hyperbolic rational homotopy Lie algebras. 
Example 5.7 Let $L$ be the evenly graded vector space with $L_{2 i}=\left\langle x_{i}\right\rangle$, a onedimensional vector space with basis element $x_{i}$ for each $i=1,2, \ldots$ Define a bracket structure on $L$ by setting $\left[x_{i}, x_{j}\right]=2(j-i) x_{i+j}$ for $i<j$. Then $L$ is a graded Lie algebra with no zero brackets. Evidently, $L$ is not a free graded Lie algebra (nor does it contain a sub-Lie algebra that is free on two generators). This example is based on the Witt algebra; we thank Simon Wadsley for pointing it out to us (via MathOverflow).

We continue to probe the $\mathrm{AF}$ conjecture, relaxing the constraint on $\mathrm{TC}_{n}(-)$. For $\mathrm{TC}_{n}\left(X_{\mathbb{Q}}\right)$ in the range $2 n-2 \leq \mathrm{TC}_{n}\left(X_{\mathbb{Q}}\right) \leq 3 n-4$ for any $n$, we are still constrained to $\operatorname{cat}\left(X_{\mathbb{Q}}\right) \leq 2$. However, in the range $2 n-1 \leq \mathrm{TC}_{n}\left(X_{\mathbb{Q}}\right) \leq 3 n-4$, we do not have $\mathrm{TC}_{n}\left(X_{\mathbb{Q}}\right)$ as low as possible, given the value of $\operatorname{cat}\left(X_{\mathbb{Q}}\right)$. The next step, then, is to consider $\mathrm{TC}_{n}\left(X_{\mathbb{Q}}\right) \leq 3 n-3$, which now allows for $\operatorname{cat}\left(X_{\mathbb{Q}}\right)=3$. We will bootstrap, using the affirmative solution to the AF conjecture for spaces of rational category 2, and establish the AF conjecture here. As with the previous result, we will actually obtain somewhat finer information.

Corollary 5.8 Suppose $X$ is a simply connected hyperbolic finite complex with $\operatorname{cat}\left(X_{\mathbb{Q}}\right)=3$ and $\mathrm{TC}_{n}\left(X_{\mathbb{Q}}\right)=3 n-3$ for some $n \geq 2$. If there exist linearly independent elements $a, b \in \pi_{\text {even }}(\Omega X) \otimes \mathbb{Q}$ with $\langle a, b\rangle=0$, and $|a| \leq|b|=2 q$, then $X_{\mathbb{Q}}^{[2 q+1]}$ is a rational co-H-space. Independently of whether or not this is the case, we have $\operatorname{cat}\left(X_{\mathbb{Q}}^{[2 r+1]}\right) \leq 2$, where $2 r$ is the lowest-degree non-zero part of $\pi_{\text {even }}(\Omega X) \otimes \mathbb{Q}$. In all cases, $X$ satisfies the $A F$ conjecture.

Proof This follows directly from part (2) of Theorem 5.4. In the second case here, we rely upon $[11 ; 3]$ to conclude the AF conjecture for $X_{\mathbb{Q}}^{[N]}$, and thus for $X_{\mathbb{Q}}$.

Returning briefly to the situation in which $\operatorname{cat}\left(X_{\mathbb{Q}}\right)=2$, and $2 n-1 \leq \mathrm{TC}_{n}\left(X_{\mathbb{Q}}\right) \leq 3 n-4$ is not as low as possible, there is one more consequence to be gleaned from Theorem 3.2. This concerns Question 5.2.

Corollary 5.9 Suppose $X$ is a simply connected hyperbolic finite complex with $\operatorname{cat}\left(X_{\mathbb{Q}}\right)=2$ and $\operatorname{TC}_{n}\left(X_{\mathbb{Q}}\right)=2 n-1$ for some $n \geq 2$. If there exist linearly independent elements $a, b \in \pi_{\text {even }}(\Omega X) \otimes \mathbb{Q}$ with $\langle a, b\rangle=0$, and $|a| \leq|b|=2 q$, then $X_{\mathbb{Q}}^{[2 q+1]}$ is a rational co-H-space.

Proof This follows from the same argument used to show part (2) of Theorem 5.4.

It is irresistible to imagine using Theorem 5.4 inductively, so as to address Question 5.2 for spaces that satisfy $\mathrm{TC}_{n}\left(X_{\mathbb{Q}}\right)=(n-1) \operatorname{cat}\left(X_{\mathbb{Q}}\right)$. Unfortunately, we are not able to do so at present. Another question prompted by our results here is the following. In principle, the hypothesis that $\mathrm{TC}_{n}\left(X_{\mathbb{Q}}\right)=(n-1)$ cat $\left(X_{\mathbb{Q}}\right)$ gives a different hypothesis on $X$ for each $n \geq 2$. However, there is some evidence to suggest they are not separate. 
Question 5.10 Does $\mathrm{TC}_{n}(X)=\operatorname{cat}\left(X^{n-1}\right)$ for some $n \geq 2$ imply that $\mathrm{TC}_{n}(X)=$ $\operatorname{cat}\left(X^{n-1}\right)$ for all $n \geq 2$ ?

\section{References}

[1] L L Avramov, Free Lie subalgebras of the cohomology of local rings, Trans. Amer. Math. Soc. 270 (1982) 589-608 MR645332

[2] I Basabe, J González, Y B Rudyak, D Tamaki, Higher topological complexity and its symmetrization, Algebraic \& Geometric Topology 14 (2014) 2103-2124

[3] R Bøgvad, C Jacobsson, Graded Lie algebras of depth one, Manuscripta Math. 66 (1989) 153-159 MR1027305

[4] O Cornea, G Lupton, J Oprea, D Tanré, Lusternik-Schnirelmann category, Mathematical Surveys and Monographs 103, Amer. Math. Soc. (2003) MR1990857

[5] A Costa, M Farber, Motion planning in spaces with small fundamental groups, Commun. Contemp. Math. 12 (2010) 107-119 MR2649230

[6] A Dranishnikov, Topological complexity of wedges and covering maps, Proc. Amer. Math. Soc. 142 (2014) 4365-4376 MR3267004

[7] M Farber, Topological complexity of motion planning, Discrete Comput. Geom. 29 (2003) 211-221 MR1957228

[8] M Farber, Instabilities of robot motion, Topology Appl. 140 (2004) 245-266 MR2074919

[9] Y Félix, S Halperin, Rational LS category and its applications, Trans. Amer. Math. Soc. 273 (1982) 1-38 MR664027

[10] Y Félix, S Halperin, J-M Lemaire, The rational LS category of products and of Poincaré duality complexes, Topology 37 (1998) 749-756 MR1607732

[11] Y Félix, S Halperin, J-C Thomas, Sur l'homotopie des espaces de catégorie 2, Math. Scand. 55 (1984) 216-228 MR787198

[12] Y Félix, S Halperin, J-C Thomas, Rational homotopy theory, Graduate Texts in Mathematics 205, Springer, New York (2001) MR1802847

[13] Y Félix, J Oprea, D Tanré, Algebraic Models in Geometry, Oxford Graduate Texts in Mathematics 17, Oxford Univ. Press (2008)

[14] Y Félix, J-C Thomas, Sur la structure des espaces de LS catégorie deux, Illinois J. Math. 30 (1986) 574-593 MR857212

[15] L Fernández Suárez, P Ghienne, T Kahl, L Vandembroucq, Joins of DGA modules and sectional category, Algebr. Geom. Topol. 6 (2006) 119-144 MR2199456

[16] M Grant, G Lupton, J Oprea, Spaces of topological complexity one, Homology Homotopy Appl. 15 (2013) 73-81 MR3117387 
[17] M Grant, G Lupton, J Oprea, New lower bounds for the topological complexity of aspherical spaces, Topol. Appl. 189 (2015) 78-91

[18] P Hilton, G Mislin, J Roitberg, Localization of nilpotent groups and spaces, Math. Studies 15, North-Holland, New York (1975) MR0478146

[19] B Jessup, A Murillo, P-E Parent, Rational topological complexity, Algebr. Geom. Topol. 12 (2012) 1789-1801 MR2979997

[20] G Lupton, J Scherer, Topological complexity of H-spaces, Proc. Amer. Math. Soc. 141 (2013) 1827-1838 MR3020869

[21] J McCleary, Homotopy theory and closed geodesics, from: "Homotopy theory and related topics", Lecture Notes in Math. 1418, Springer, Berlin (1990) 86-94 MR1048178

[22] Y B Rudyak, On higher analogs of topological complexity, Topology Appl. 157 (2010) 916-920 MR2593704

[23] Y B Rudyak, Erratum to “On higher analogs of topological complexity" [Topology Appl. 157 (2010) 916-920], Topology Appl. 157 (2010) 1118 MR2593724

[24] W Singhof, On the Lusternik-Schnirelmann category of Lie groups, Math. Z. 145 (1975) 111-116 MR0391075

[25] G H Toomer, Topological localization, category and cocategory, Canad. J. Math. 27 (1975) 319-322 MR0370571

[26] G W Whitehead, Elements of homotopy theory, Graduate Texts in Mathematics 61, Springer, New York (1978) MR516508

MG: Institute of Pure and Applied Mathematics, University of Aberdeen Fraser Noble Building, Aberdeen, AB24 3UE, UK

GL, JO: Department of Mathematics, Cleveland State University 2121 Euclid Avenue, Cleveland, $\mathrm{OH} 44115$, USA

mark.grant@abdn.ac.uk, g.lupton@csuohio.edu, j.oprea@csuohio.edu

Received: 29 April $2014 \quad$ Revised: 21 October 2014 